\title{
Fast Iris Localization Based on Image Algebra and Morphological Operations
}

\author{
Ashwaq T. Hashim \\ Zina A.Saleh \\ Control and Systems Eng. Dept., Technology University, Baghdad, Iraq \\ 60102@uotechnology.edu.iq 61266@student.uotechnology.edu.iq
}

Submission date:- 1/9/2018 Acceptance date:- 26/9/2018 Publication date:- 27/1/2019

Keywords: Iris localization; Arithmetic operations; Morphological operators; iris biometrics.

\begin{abstract}
The localization of the iris is the most significant factor in biometrics of the iris, which traditionally assumes strictly controlled environments. The proposed method includes the pupillary and limbic iris boundaries localization. A primary advantage of image arithmetic operations is that the process is straightforward and therefore fast, these characteristics are employed and combined with the morphological operators in the designing of the proposed algorithm. The proposed algorithm takes into account the noise area which is found in various parts of the eye image such as light reflections, focus and small visible iris. The experimental results are conducted on a collection of iris images consist of 756 images belong to Chinese Academy of Sciences Institute of Automation (CASIA V-1) and 450 images belong to Multi Media University (MMU V-1) databases. The results indicate a high level of accuracy using the proposed technique. Moreover, the comparison results with the state-ofthe-art iris localization algorithms expose considerable improvement in segmentation accuracy while being computationally more efficient.
\end{abstract}

\section{Introduction}

For the automated personal identification, iris recognition is one of the most accurate and broadly employed methodology. A typical iris recognition system comprises segmentation, normalisation, feature extraction and matching. In an excellent iris authentication system, the iris segmentation is a very significant part, which must be able to cope with many adverse circumstances such as the blurred outer border of iris, light reflex points in pupil, eyelids and effected eyelashes [1]. However, traditional iris localization methods and matching features depend only on near-infrared illumination and require samples to be taken under strictly restricted conditions [2], which is the main difficulty of deploying an iris recognition system in civilian and surveillance applications. Several ways to address the problem were suggested in the literature. The accuracy and computation time for currently available iris localization algorithms are still below the expectations and requires further improvement for the deployments. Therefore, the proposed method was designed to take the advantage of the image arithmetic operations features with morphological operators features for localizing the iris.

This paper is organised as follows: Section 2 overviews the existing literature for iris localization. The proposed method for iris localization is explained in section 3 . The experimental results of the proposed system are provided in section 4 , and final conclusions are shown in section 5.

\section{Literature Review}

The localization of the iris represents the core of iris recognition system. Most iris localization is based on techniques proposed by Daugman [3], Wildes [4] and active contour models [5].

Daugman's integro-differential operator [6],[7],[8] searches the image domain for the maximum in the blurred partial derivative, concerning the increasing radius $\mathrm{r}$ of the normalised contour integral of $I(x, y)$ along with a circular arc $d s$ of radius $r$ and centre coordinates $\left(x_{0}, y_{0}\right)$. In Eq. (1), $\mathrm{G}_{\sigma}(r)$ is a smoothing function such as a Gaussian of scale $\sigma$. The full operator executes a frequent search for a maximal contour integral derivative with increasing radius in successive accurate scales of analysis through the three parameter centre space coordinates and radius $\left(r, x_{0}, y_{0}\right)$ determining the contour integration path [3].

$$
\max _{\left(r, x_{0}, y_{0}\right)}\left|G_{\sigma}(r) * \frac{\partial}{\partial_{r}} \oint_{r, x_{0}, y_{0}} \frac{I(x, y)}{2 \pi r} \mathrm{ds}\right|
$$

Despite its high efficiency, Daugman's operator does not always segment the iris accurately when the input image of the iris is noisy. This noise can be in the form of a reflection, or that the eye in the image is not fully open 
or the eyelashes covering part of the iris area. Low-quality iris images cause a poor recognition performance as long as the traditional processing of iris images is performed

On the other hand, Wildes proposes the circular Hough transform. In this method, the boundary is estimated by rediscovering the circular structures within the binary edge map of the points $(x j, y j), j=1, . ., n$, over the image gradient. The goal is to find a triplet of two-dimensional origin coordinates and radii that maximises the expression $\mathrm{H}$ (xc, yc, r) in Eq. (2) [4]:

$$
H\left(x_{c}, y_{c}, r\right)=\sum_{i=1}^{n} h\left(x_{j}, y_{j}, x_{c}, y_{c}, r\right)
$$

where $h\left(x_{j}, y_{j}, x_{c}, y_{c}, r\right)=1$ when $\left(x_{j}-x_{c}\right)^{2}+\left(y_{j}-y_{c}\right)^{2}-r^{2}=0$.

The appropriate majority methods are edge-based methodologies [9-15], at first; the edge features are utilised to detect the outer and inner iris borders. Images are taken under the visible wavelength spectra but in uncooperative environments of recognition might be lead to light reflections and specular [16], which could produce false edges in edge maps. Beside, eyelashes, poor focus and blurring motion might destroy the edges. Significant experience exposes that the techniques which have been depending on the edge-based detection are susceptible to these false edges. Secondly, the Hough transform is computationally exhaustive through the "brute force" approach, and thus may not be suitable for real-time applications.

Active contour models [5], [17], [18] provide flexible contours that allow the iris and eyelid boundaries to be detected at the same time, but the performance of approaches based on active contour models may be downgraded due to reflections, the heterogeneous of the iris and unclear boundaries, and using such models needs also sensibly of many parameters to get a suitable curve, furthermore these models need computation time complexity.

\section{Proposed Iris Localization Algorithm}

The iris of the human is an annular part of a pupil that represents the inner border and the white sclera that represents the outer border. An approach of iris localization algorithm on the basis of image arithmetic operations and morphological operators is proposed. Two main stages are featured in the proposed algorithm, namely iris localization (i.e., Detection of the outer boundary of the iris region and pupil localization (i.e., Detection of the inner boundary of the pupil region).

\subsection{Pupil Localization}

For the detection of the inner circle of the iris, the behaviour of the intensity of the image is taken into consideration in each of the pupil and iris parts of the eye. The overall intensity of the pupil's area is relatively smaller than that of the other, from the whole eye image. Next, the most significant dark and packed part that will appear in the image of the eye representes the pupil. The approximate pupil center is discovered by calculating the center of iris region mass in eye image after getting the benefit of these attributes. The following steps are applied to Localize pupil:

\section{Algorithm 1: Pupil Localization}

\section{Step1: (Apply image addition)}

Because of the features that the pupil owns them such as darkness, large connected, and packed area, thus these features are exploited by image adding operation to increase overall image brightness which is eliminated the most details of the image but it still preserved the area of the pupil. Image addition is applied consecutively using the following formula:

$$
g(x, y)=\sum_{i=0}^{T} f(x, y)
$$

Where $\mathrm{g}(\mathrm{x}, \mathrm{y})$ output pixel values, $f(\mathrm{x}, \mathrm{y})$ the input pixel values, $T$ the threshold value which is selected using Otsu global image threshold method [19]. The Otsu's method chooses the threshold to reduce the intraclass variance of the thresholded black and white pixels. According to this method, the level representing the value of a normalised intensity is located in the range $[0,1]$. The $T$ is computed using the following formula:

$$
T=\text { Round }(\text { level } \times 10)
$$

Figure (1) shows the result of this step. 


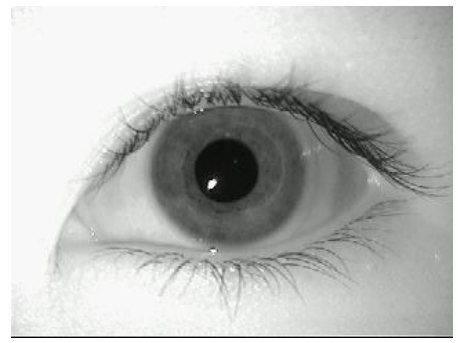

(a)

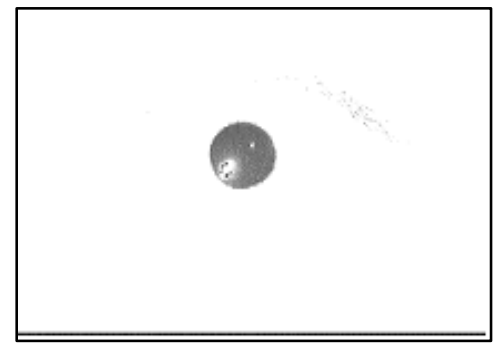

(b)

Fig.1: Consecutive image addition. (a) Original image (b) Resulted image with $T=7$.

\section{Step2: (Cut boundaries)}

The image of the eye might hold dark, thick eyebrows, thus to avoid these pixels, considered as pupil pixels (the first top rows, last rows, first left columns, and first right columns) are excluded with a specific exclusion rate (ER). For example, when the ER is set to 20 , the first $20 \%$ of the first rows and the last $20 \%$ of the eye image rows are excluded from the domain of the seed point scan. Also, the pixels which fall within $20 \%$ of the first left columns and $20 \%$ of the last right columns are omitted as depicted in Figure (2).

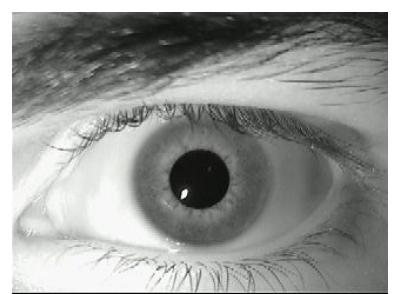

(a)

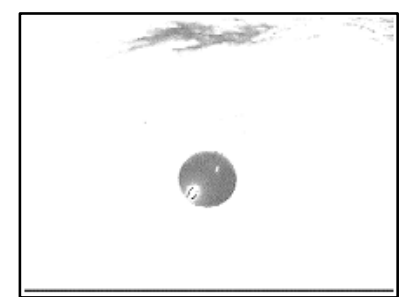

(b)

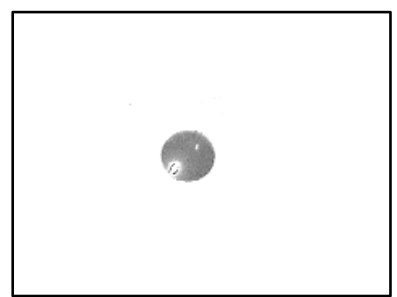

(c)

Fig.2: Cut Boundaries. (a) Original image, (b) After Concussive image addition, (c) image after excluding ER=20\% from rows and columns

Step3: (Convert to binary)

The resulted image is converted to binary using the following formula:

$$
\operatorname{Binary}(\mathrm{x}, \mathrm{y})= \begin{cases}1 & \text { if } g(x, y)=255 \\ 0 & \text { if } g(x, y)<>255\end{cases}
$$

Where $g(x, y)$ is the intensity value at location (x, y), and $\operatorname{Binary}(x, y)$ is the converted pixel value. Figure (3) depicts the converted binary image.

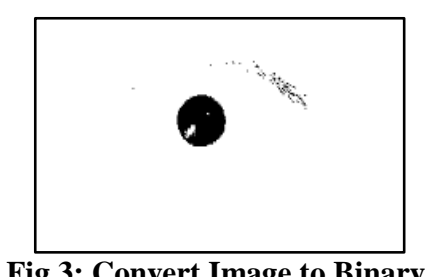

Fig.3: Convert Image to Binary.

\section{Step4: (Removal of Reflection Points)}

As notice is that the MMU-1 database includes white points in the region of the pupil. To eliminate existing points of reflection, the region filling process is applied to the binary images to detect these points. The following substeps are used to reduce reflex points:

Step4.1: $A$ is an input binary image and $\beta(\mathrm{A})$ its boundary.

Step4.2: Let $X_{0}=\mathrm{P} \quad / / \mathrm{P}$ is a point inside $\beta$ (A) (i.e., a non-boundary pixel)

Step4.3: While $X_{k}<>X_{k-1}$ do $X_{k}=\left(X_{k-1} \oplus \mathrm{B}\right) \cap \mathrm{A}^{\mathrm{C}} \quad / /$ where $\mathrm{B}$ is given a symmetric //structuring element 
Step4.4: $\mathrm{X}_{\mathrm{F}}=\mathrm{X}_{\mathrm{k}} \cup \mathrm{A}$

The fill reflex region in the image is shown in Figure (4).

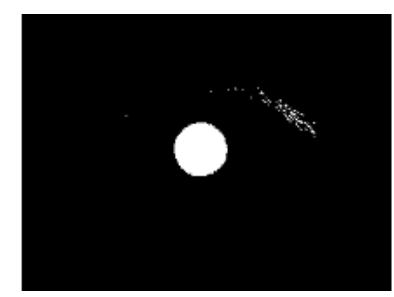

Fig.4: Removal of Reflection Points.

\section{Step5: (Cleaning Image)}

To remove the small detail remaining in the produced binary image, two steps are performed:

Step5.1: Filtered image using a median filter.

Step5.2: Then applied the morphological erosion operation. The erosion operation is implemented by taking a $3 \mathrm{x}$ 3 window, then the centre of the structure is checked if its value is 0 , after that its 8 -neighbours is checked; if at least three pixels contain the value 1, the value of the centre is replaced to 1. Cleaning image is shown in Figure (5).

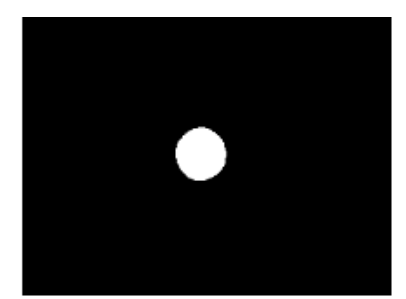

Fig.5: Cleaning an image.

\section{Step6: (Collect Pupil Region)}

The region growing method is used to collect the pupil pixels, and then the coordinates $\mathrm{x}$ and $\mathrm{y}$ of these collected pixels are stored. The growing part is shown in following substeps:

Step6.1: set a List to the seed point

Step6.2: While (List not empty)

$$
\begin{aligned}
& \text { Begin } \\
& \text { pixel }=\text { next pixel from List }
\end{aligned}
$$$$
\text { set Pixel(pixel) }
$$$$
\text { For (each of neighbor's pixel) }
$$$$
\text { Begin }
$$

If (neighbor is within region and neighbor not set)

Store pixel coordinate $\mathrm{x}, \mathrm{y}$ and insert neighbour to List

End If

End While

End For

\section{Step7: (Calculate Pupil Center)}

Compute the pupil centre $\left(x_{\mathrm{p}}, \mathrm{y}_{\mathrm{p}}\right)$ by taking the average of coordinates $(\mathrm{x}, \mathrm{y})$ of the points that fall within the pupil region using the following equations:

$$
\begin{aligned}
& X_{p}=\frac{1}{N} \sum_{i=1}^{N} X_{i} \\
& Y_{p}=\frac{1}{N} \sum_{i=1}^{N} Y_{i}
\end{aligned}
$$

Where $N$ is the point numbers of the pupil region that are collected.

\section{Step8: (Calculate the Pupil Radius)}

From the centre point $\left(x_{p}, y_{p}\right)$, moves in the four directions (i.e., left, right, up and down) and sets the first white pixel on each side. $X_{L}, X_{R}, X_{T}$, and $X_{B}$ are the first white pixels to the left, right, bottom, and top sides 
respectively, when scanning horizontally by $\mathrm{x}=\mathrm{x}_{\mathrm{p}}$ and scanning vertically by $\mathrm{y}=\mathrm{y}_{\mathrm{p}}$. The horizontal and vertical pupil radiuses $R_{h}$ and $R_{v}$ are then calculated as follows:

$$
\begin{aligned}
& R_{h}=\frac{1}{2}\left(X_{L}-X_{R}\right) \\
& R_{v}=\frac{1}{2}\left(X_{T}-X_{B}\right)
\end{aligned}
$$

Figure (6) shows the computed four directions in pupil region.

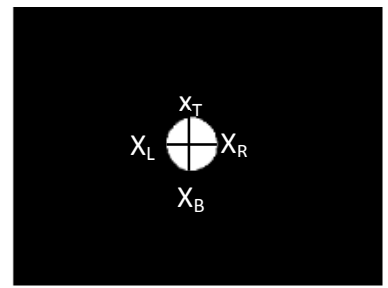

Fig.6: Four directions of the pupil region.

\subsection{Iris Localization}

The next stage involves isolating the iris of the eye, and this stage included the following consecutive steps: Step1: (Eye Image Enhancement)

In order to make the iris boundaries more apparent, initially it will be apply mapping process represented by histogram stretching on the original eye image, by applying the following substeps:

Step1.1: Use stretchlim(I) which is returns the Low and High pixel values that specify lower and upper limits that can be used for contrast stretching eye image.

Step2.1: Adjust the contrast in the eye image using Low and High pixel values to set the limits. Figure (7) depicts the result of this step.
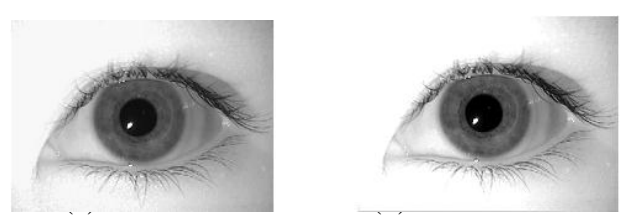

Fig.7: Eye Image Enhancement. (a) Original image. (b) Enhanced image.

\section{Step2: (Smoothing of Image)}

The produced enhanced image is smoothed by using mean filter with widow size $9 \times 9$. The smoothed image $S$ is depicted in Figure (8).

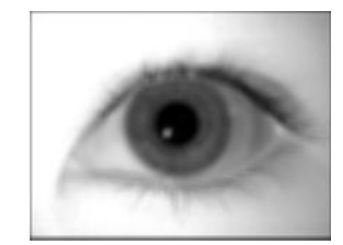

Fig.8: The smoothed Image.

\section{Step3: (Finding Threshold Value)}

The threshold value is selected depending on the relationships between the characteristic of the original image and the image after thresholding with a small value to preserve the dark pixels only and then performed the following substeps:

Step3.1: Compute the mean $\left(m_{l}\right)$ and standard deviation $\left(\sigma_{1}\right)$ of the original eye image.

Step3.2: Calculate the mean $\left(m_{2}\right)$ and standard deviation $\left(\sigma_{2}\right)$ of the eye the image after thresholding with a small value (i.e., 0.15).

Step3.3: Compute the threshold value using the following formula:

$$
T_{1}=m_{2} \times \sigma_{2}+m_{1} \times \sigma_{1}
$$

\section{Step4: (Multiply Image)}

In this step, the smoothed image $S$ has been multiplied by threshold value $T_{1}$. Figure (9) shows the image after multiplying by $T_{l}=0.4$.

$$
M=S \times T_{1}
$$




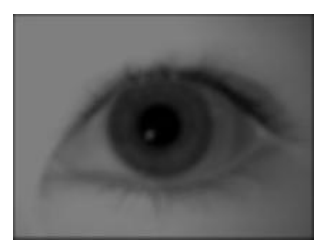

Fig.9: Multiplied Image by Threshold $T_{1}=0.4$

\section{Step5: Convert to Binary}

The Resulted Image is converted to binary using the following formula:

$$
B(\mathrm{x}, \mathrm{y})= \begin{cases}0 & \text { if } M(x, y)<T H \\ 1 & \text { if } M(x, y) \geq T H\end{cases}
$$

Where $M(x, y)$ is the intensity value of image $M$ at location (x, y), and $B(x, y)$ is the intensity value of converted image $B$ at location (x,y). The $T H$ is the threshold value which is an experimental value. Figure (10) shows the transformed image with $T H=60$.

\section{Step6: Clean image}

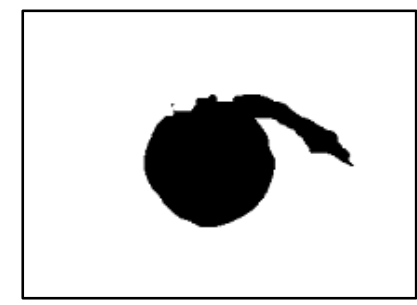

Fig.10: Converted image by $T H=60$.

Removing all connected components (objects) that have fewer than $P$ (i.e.,50) pixels from the binary image, producing another binary image. The default connectivity is 8 . Then, erosion operation is performed.

\section{Step7: Compute the Radius}

Step7.1: Horizontal scan is applied from the centre of the pupil to the left and right until reaching the first white pixels from two directions, then set $R_{1}$ and $R_{r}$ to the number of pixels that are scanned in these directions.

Step7.2: In some cases, part of pupil is covered by the eyelashes and the arcs of the upper border of left or right eye continue with the iris area so that the scanning will go far away from the border of iris to overcome these cases, the following condition are executed:

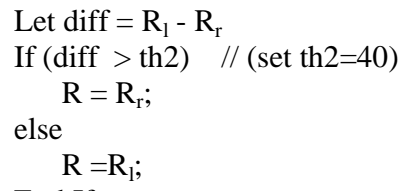

End If

Step7.3: The iris radius is equal to:

$$
\text { R_iris }=\mathrm{R}-\mathrm{R}_{\mathrm{h}}
$$

\section{Experimental Results}

The proposed system was implemented in Matlab (R2015a) on a windows operating system with Core i5 PC of $2.67 \mathrm{GHz}$ processor and $4 \mathrm{~GB}$ DRAM. The proposed iris segmentation method performance was tested using MMU-V1 and CASIA V-1 databases. The CASIA V-1 iris image database [20] includes 756 "non-ideal" images of 108 different users. Each image of the iris is grayscale with $320 \times 280$ pixels resolution. The MMU V-1 iris database [21] contains 450 images of 45 people captured using the LGIrisAccess®2200 camera. This semiautomatic camera operates on over 7-25 cm. The produced iris images are belong to 100 volunteers of various ages and nationality. For each eye image of a person has 5 iris images. And a resolution for each image is of $320 \times 240$ pixels in grayscale. Figure (11) shows examples of the two famous databases. 


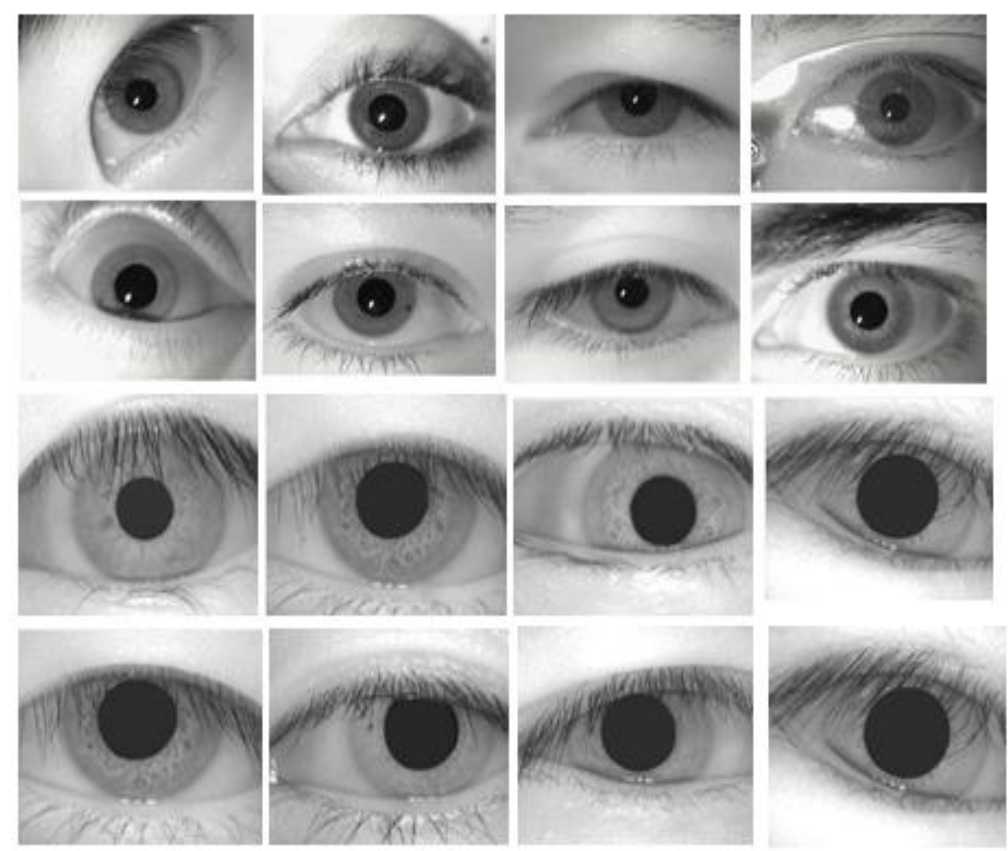

Examples of the correct pupil and iris localization in which the proposed method yields high-accurate results in the existence of various noises which have been listed in Figure (12) and (13) respectively.
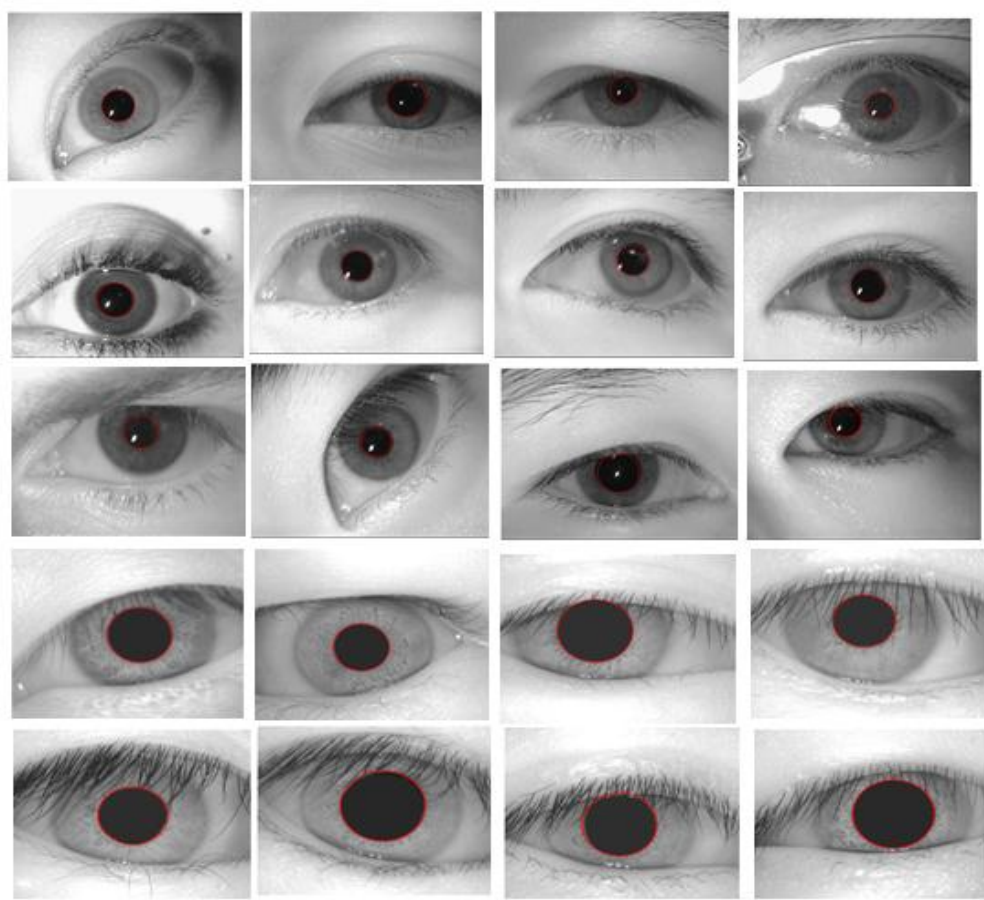

Fig.12: Samples of accurate pupil localization of images for MMU-1 and CASIA-1 databases. 


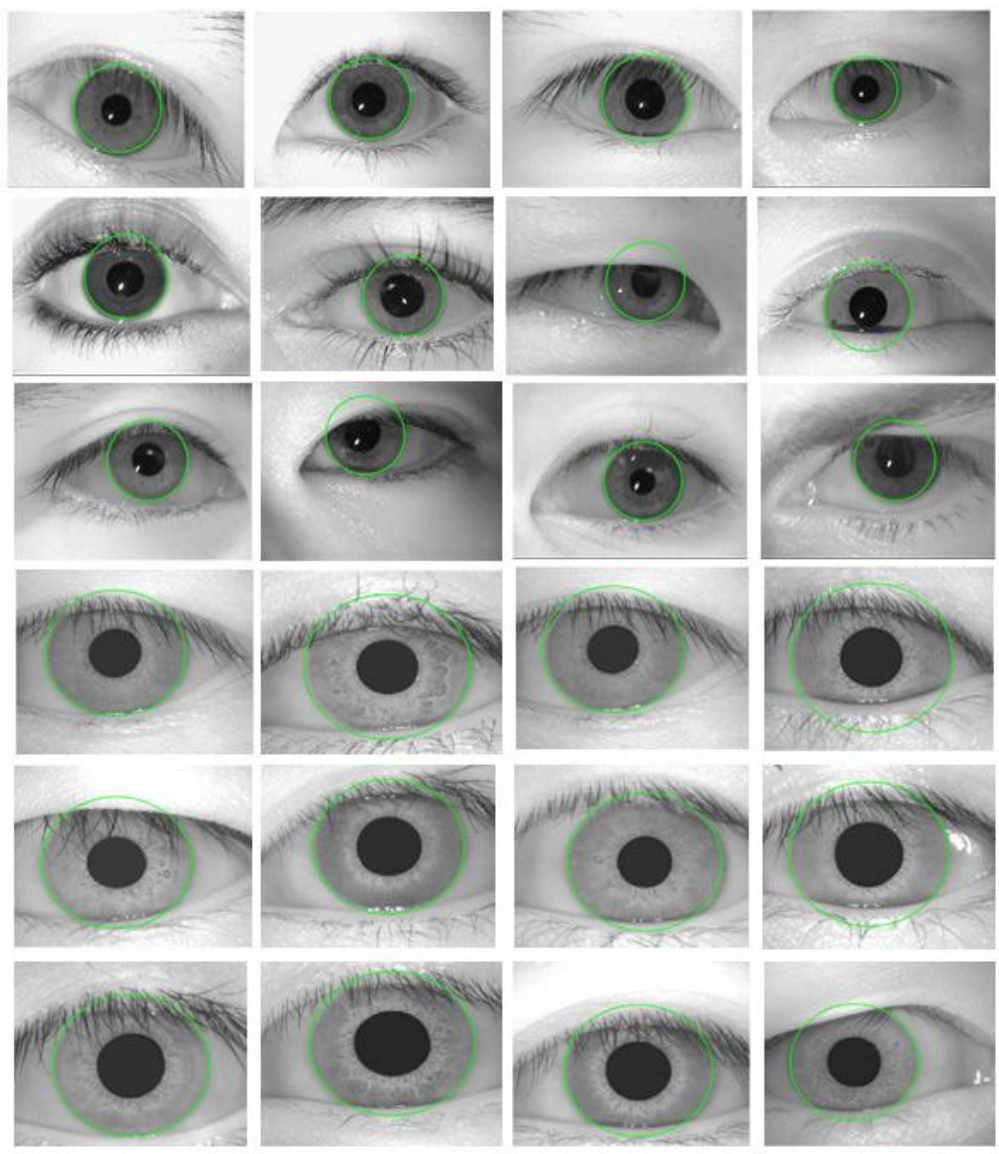

Fig.13: Samples of accurate iris localization of images for MMU-1 and CASIA-1 databases

The proposed iris localization algorithm was compared with the well-known iris segmentation algorithms introduced by Daugman [3], Wildes [4] and Libor Masek [9]. Further to some of previous iris localization algorithms proposed by Liam and Chekima [22], Guang-zhu XU et al. [23], Weiqi Yuan et. al. [24], Muhammad et al. [25] and Suhad et. al. [26]. Daugman's [3], Wildes's [4] is implemented in this work and the code provided by Libor Masek is used [27] to Libor Masek's algorithm [9]; while iris localization results of Chekima's, Guangzhu's, Weiqi Yuan's, Muhammad's, Suhad's algorithms were taken from the publishing works [28], [29], [30]. Table 1 and Table 2 show the results of the proposed algorithm accuracy with compared to other algorithms. Where the segmentation accuracy is calculated according to the equation as below:

$$
\text { Accuracy }=\frac{\text { Number of correctly segmented images }}{\text { Total number of images }} \times 100
$$

From the results listed in Table 1 and Table 2, it is noted that the proposed method based on image algebra and morphological operators has the best performance. The accuracy rate bellowing to MMU-1 is up to $98.78 \%$. (i.e., 99.33 for pupil localization and 98.22 for iris localization) and $100 \%$ belong to CASIA-1. The failure reason for the proposed pupil detection is that the eyelashes have interfered with the pupil area. Thus, the result of the consecutive addition will eliminate some part of the pupil when the intensity of the eyelashes are different from the pupil as shown in figure (14). 
Table 1: Comparisons of iris localization accuracy for MMU-1 database

\begin{tabular}{|l|c|c|}
\hline Methodology & \multicolumn{2}{|c|}{ Accuracy rate } \\
\hline Daugman & MMU1 & $55.22 \%$ \\
\hline Wiles & MMU1 & $93.33 \%$ \\
\hline Masek & MMU1 & $95.66 \%$ \\
\hline Guang-zhu XU et. al. & MMU1 & $98.42 \%$ \\
\hline Weiqi Yuan et. al. & MMU1 & $99.45 \%$ \\
\hline Suhad Ali et. al. & MMU1 & $97.5 \%$ \\
\hline Proposed method & MMU!-1 & $98.78 \%$ \\
\hline
\end{tabular}

Table 2: Comparisons of iris localization accuracy for CASIA-1 database

\begin{tabular}{|c|c|c|}
\hline Methodology & \multicolumn{2}{|c|}{ Accuracy rate } \\
\hline Daugman & CASIA-1 & $54.44 \%$ \\
\hline Wiles & CASIA-1 & $86.49 \%$ \\
\hline Masek & CASIA-1 & $83.92 \%$ \\
\hline Liam and Chekima & CASIA-1 & $64.64 \%$ \\
\hline Muhammad Talal et. al. & CASIA-1 & $99.47 \%$ \\
\hline Suhad Ali et. al. & CASIA-1 & $99.85 \%$ \\
\hline Proposed method & CASIA-1 & $100 \%$ \\
\hline
\end{tabular}

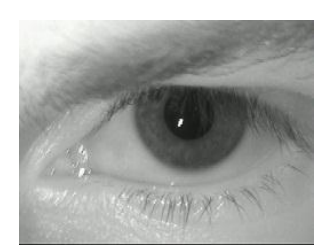

(a)

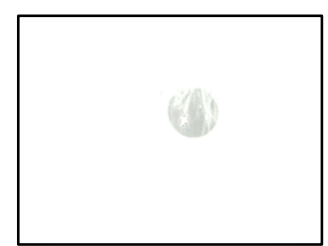

(b)

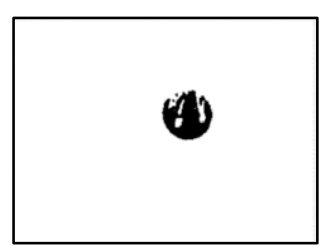

(c)

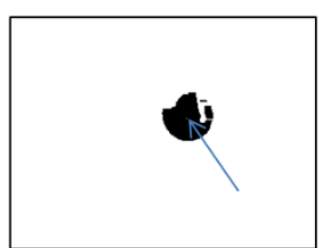

Computed center

(d)

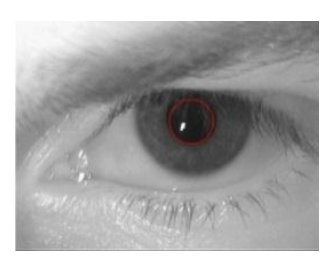

(e)

Fig.14: Inaccurate pupil localization. (a) Thomas left eye from MMU-1 (b) Consecutive image addition. (b) Cleaning and convert to binary. (d) After region filling (e) Localized pupil.

The failure reason for the proposed iris detection due to the value of threshold $T_{l}$ which is obtained from eq.(10). For Tanwn left eye, the computed $T_{1}$ is 0.39 which is led to failing result of iris localization. When the threshold is adjusted to $T_{l}=0.42$, the iris is localized correctly as shown in Figure (15). 


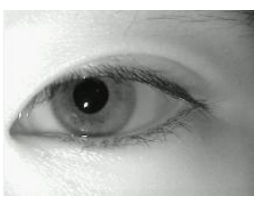

(a)

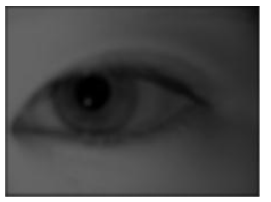

(b)

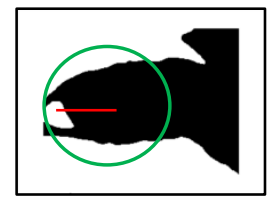

(c)

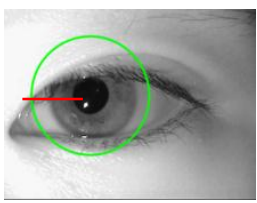

(d)

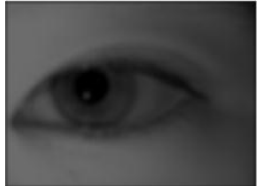

(e)

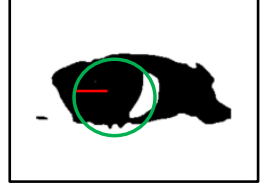

(f)

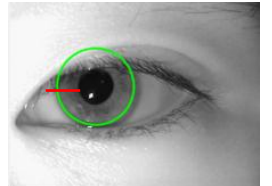

(g)

Fig.15: Inaccurate iris localization. (a) Tanwn left eye from MMU-1. (b) Multiplied eye image by threshold $T_{1}=0.39$. (b) Converted image by $T H=60$. (d) Localize iris (e) Multiplied eye image by threshold $T_{1}=0.42$. (f) Transformed image by $T H=60$. (g) Localize iris.

Table 3 shows a time comparison among the proposed methodology and the three most commonly used algorithms for localising the iris, [3], [4] and [9]. Because that Daugman, Wildes and Masek techniques required a comprehensive search that results in a very high cost of computation, So that, the proposed method employed the arithmetic operators which is very simple and therefore fast.

Table 3: Computation time comparison of proposed method with some other methods used for iris localization

\begin{tabular}{|c|c|}
\hline Name of method & $\begin{array}{c}\text { Computation } \\
\text { time(Sec.) }\end{array}$ \\
\hline Daugman & 13.38 \\
\hline Wiles & 7.35 \\
\hline Masek & 5.21 \\
\hline Proposed method & 0.02 \\
\hline
\end{tabular}

\section{Conclusions}

The image arithmetic operations are elementary and therefore very fast consequently these features are exhausted to design a quick method to localize iris. When the image is added to itself, the resulted image will be bright. Further, if the image continues with addition several times this lead to disappear all details of image accept the parts which are very dark such as pupil, eyelashes and eyebrow. The number of addition must be chosen carefully due to the eye images difference in its brightness. Therefore, the threshold value is selected based on Otsu global image threshold method. Another threshold was required in the proposed method for iris localization, which was selected depending on the statistical relationships between of the original image and the image after thresholding with a small value to preserve only the dark pixels. This value is useful for highlighting the iris area after multiplying the iris image by a threshold value which is wanted to identify. Most of the previous methods depended on techniques using the Hough Transform, active contour models or integro-differential operator. All these methods require a comprehensive search that results in a very high cost of computation. The proposed method was applied on CASIA V-1, and MMU V-1 iris image databases. The results showed that the proposed iris localization method outperforms over some state-of-the-art iris localization methods in both segmentation accuracy and computation time. 


\section{References}

[1] J.K. Pillai, V.M. Patel, R. Chellappa, and N.K . Ratha, "Secure and robust iris recognition using random projections and sparse representations", IEEE Transactions on Pattern Analysis and Machine Intelligence, vol. 33 no. 9, pp.1877-1893, 2011.

[2] C. Tan, and A. Kumar, "Unified framework for automated iris segmentation using distantly acquired face images", IEEE Transactions on Image Processing, vol. 21, no. 9, pp.4068-4079, 2012.

[3] J.G. Daugman, "High confidence visual recognition of persons by a test of statistical independence", IEEE Transactions on Pattern Analysis and Machine Intelligence, vol. 15, no. 11, pp.1148-1161, 1993.

[4] R.P. Wildes, "Iris recognition: an emerging biometric technology", Proceedings of the IEEE, vol. 85, no. 9, pp.1348-1363, 1997.

[5] M. Kass, A. Witkin, and D.Terzopoulos, "Snakes: Active contour models", International Journal of Computer Vision, vol. 1, no.4, pp.321-331, 1988.

[6] V. Kumar, A. Asati, and A. Gupta, "Iris localization based on integro-differential operator for unconstrained infrared iris images", Signal Processing, Computing and Control (ISPCC), 2015 International Conference on, pp. 277-281, 2015.

[7] A. Radman, K. Jumari, and N. Zainal, "Fast and reliable iris segmentation algorithm", IET Image Processing, vol. 7, no. 1, pp.42-49, 2013.

[8] Q. Wang, Z. Liu, S. Tong, Y. Yang, and X. Zhang, "Efficient iris localization via optimization model", Mathematical Problems in Engineering, 2017.

[9] L. Masek, "Recognition of human iris patterns for biometric identification", B.Sc. thesis, the School of Computer Science and Software Engineering, The University of Western Australia, 2003.

[10] Y. Chen, M. Adjouadi, , C. Han, J. Wang, A. Barreto, N. Rishe, and J. Andrian, "A highly accurate and computationally efficient approach for unconstrained iris segmentation", Image and Vision Computing, vol. 28, no.2, pp.261-269, 2010.

[11] L.L. Ling, and D.F. de Brito, "Fast and efficient iris image segmentation", Journal of Medical and Biological Engineering, vol. 30, no. 6, pp. 381-392, 2010.

[12] A. Uhl, and P. Wild, "Weighted adaptive hough and ellipsopolar transforms for real-time iris segmentation", In Proceedings of the 5th IEEE International Conference on Biometrics, New Delhi, India, pp. 283-290, 29 March-1 April 2012.

[13] J.J. Fernandez, and A. Mathew, "Irregular pupil localization using connected component analysis", Automation, Computing, Communication, Control and Compressed Sensing (iMac4s), 2013 International Multi-Conference on, pp. 155-159, 2013.

[14] Z. Zhao, and A. Kumar, "An accurate iris segmentation framework under relaxed imaging constraints using total variation model". In Proceedings of the IEEE International Conference on Computer Vision, Santiago, Chile, pp. 3828-3836, 7-13 December 2015.

[15] Z. Li, "An iris recognition algorithm based on coarse and fine location", Big Data Analysis (ICBDA), 2017 IEEE 2nd International Conference on, pp. 744-747, 2017.

[16] G. McConnon, F. Deravi, S. Hoque, K. Sirlantzis, and G. Howells, " A survey of point-source specular reflections in noisy iris images", Emerging Security Technologies (EST), 2010 International Conference on, pp. 13-17, 2010.

[17] E. Ouabida, A. Essadike, and A. Bouzid, "Optical approach for iris segmentation and tracking", Information Science and Technology (CiSt), 2016 4th IEEE International Colloquium on, pp. 476-480, 2016.

[18] M.A.M. Abdullah, S.S. Dlay, W.L. Woo, and J.A. Chambers, "Robust iris segmentation method based on a new active contour force with a noncircular normalization", IEEE Trans. Syst. Man Cybern, PP. 1-14, 2017.

[19] N. Otsu, "A threshold selection method from gray-level histograms", IEEE Transactions on Systems, Man, and Cybernetics, vol. .9, no. 1, pp.62-66, 1979.

[20] CASIA, (2003), CASIA iris image database (ver. 1.0) http://www.sinobiometrics.com

[21] Multimedia University, Iris database, http://www.persona.mmu.edu.my/

[22] L.W. Liam, A. Chekima, L.C. Fan, and J.A. Dargham, "Iris recognition using self-organizing neural network", Research and Development, SCOReD Student Conference on, pp. 169-172, 2002.

[23] G. Xu, and Z. Zhang, "A novel and efficient method for iris automatic location", Journal of China University of Mining and Technology, vol. 17, no. 3, pp.441-446, 2007. 
[24] W. Yuan, Z. Lin, and L. Xu, "A rapid iris location method based on the structure of human eyes", IEEEEMBS 2005, Engineering in Medicine and Biology 27th Annual Conference, Shanghai, China, pp. 3020-3023, September 1-4, 2005.

[25] M. T. Ibrahim, T. Mehmood, M.A. Khan, and L. Guan, "A novel and efficient feedback method for pupil and iris localization", International Conference Image Analysis and Recognition, pp. 79-88, 2011.

[26] S. A. Ali, and L. E. George, "New method for iris localization for personal identification", International Conference on Information Technology in Signal and Image Processing-itSIP, India, 2013.

[27] L. Masek, and P. Kovesi, "Matlab source code for a biometric identification system based on iris patterns", Bachelor (B.S.) project, The School of Computer Science and Software Engineering, The University of Western Australia, 2003.

[28] H. Proença, and L.A. Alexandre, "UBIRIS: A noisy iris image database", International Conference on Image Analysis and Processing, pp. 970-977, 2005. http://iris.di.ubi.pt

[29] I. A. Saad, and L. E. George, "Robust and fast iris localization using contrast stretching and leading edge detection", International Journal of Emerging Trends \& Technology in Computer Science (IJETTCS), vol. 3, no. 2, pp.61-67, 2014.

[30] S. A. Ali, and L. E. George, "New Approach of Iris Localization for Personal Identification", International Conference on Information Technology in Signal and Image Processing (ITSIP-2013), Elsevier, 2013.

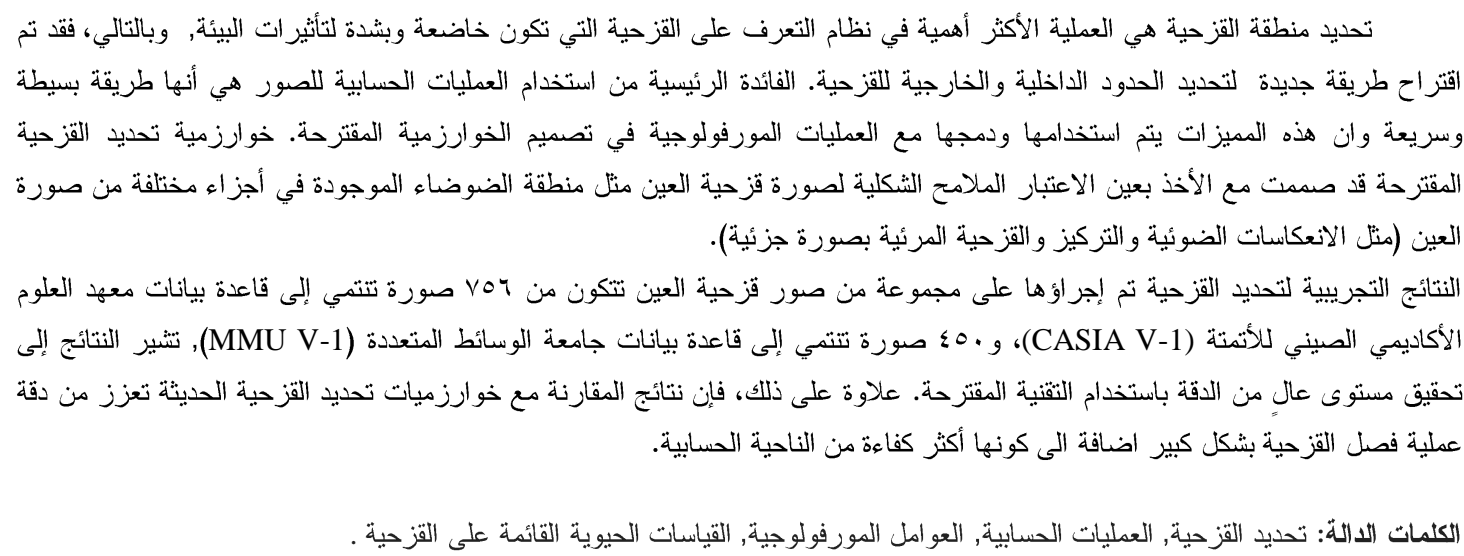

